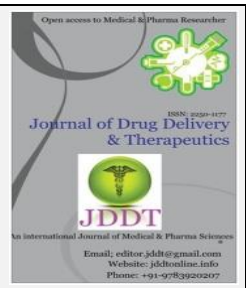

Open $\odot$ Access

Review Article

\title{
Good Statistical Practice: A Key To Effective and Safe Drug Product
}

\author{
R. Santosh Kumar and G. Saisandeep Naidu \\ GITAM Institute of Pharmacy, GITAM (Deemed to be University), Rushikonda, Visakhapatnam, 530045.
}

\begin{abstract}
In the pharmaceutical R\&D the evaluation procedures play a key role in developing a potent graded product focusing on the various tech nical advancements it's easy to overcome hazards. Emphasis is placed on the product's development to reach specifications of USFDA, US Pharmacopeia, National Formulary for achieving standards that provide insight on evaluation techniques of drug formulation. Adoption of guidance's and guidelines cited will strive for achieving good laboratory practice(GLP) good clinical practice(GCP) and good manufacturing practice(cGMP).Embracing statistical approach provides accuracy and reliability of results.
\end{abstract}

Keywords: Good Statistical Practice, Randomization, Sample

Article Info: Received 14 June 2019; Review Completed 17 August 2019; Accepted 22 August 2019; Available online 30 August 2019

\section{Cite this article as:}

Santosh Kumar R, Saisandeep Naidu G, Good Statistical Practice: A Key To Effective and Safe Drug Product, Journal of Drug Delivery and Therapeutics. 2019; 9(4-s):806-808 http://dx.doi.org/10.22270/jddt.v9i4-s.3369

*Address for Correspondence:

R. Santosh Kumar, GITAM Institute of Pharmacy, GITAM (Deemed to be University), Gandhinagar, Visakhapatnam530045, Andhra Pradesh, INDIA.

\section{INTRODUCTION:}

Good statistics practice (GSP)is the set of principles or the standard operating procedures_to validate the (or) conduct, analysis, evaluation of the pharmaceutical product at various stages of it's research and development (R\&D).

These procedures or principles help out to minimize the bias and variability that occur before during or after the product development the concept of GSP has raised forward by relating or following the standards and specifications raised by the USFDA and formularies and other regulatory bodies, for example the us regulatory requirements for drug research and development aides by the pharmacopeia and national formulary which includes set of procedures ,sampling techniques and acceptance criteria for the development of a pharmaceutical product.

The guidelines and guidance will help out for achieving the standards of good manufacturing practices(GMP)good clinical practice(GCP) and current good manufacturing practice(cGMP),Recently the ICH have outlined the concept of GSP on statistical principles for clinical trials that not only provide accuracy \& reliability but also validity and integrity of the studies.

\section{ROLE OF STATISTICS IN DRUG DEVELOPMENT:}

During the process of drug research and development (R\&D) statistics are necessarily applied at various stages to meet regulatory requirements for the effectiveness, safety, identity, purity, of the drug product under investigation. These includes a hectic process that involves screening of wide number of compounds in order to differentiate \& identify the various potencies of the compound required for the formulation of the drug product.

As the process involves following the guidelines aided by FDA and IND regarding the chemistry manufacturing and control (CMC) of the drug product that provides the identity of the drug and differentiate it in terms of efficacy kinetics and pharmacological aspects of drug in the intrinsic environment.

The drug statistics are applied in order to validate a developed analytical method or to determine the expiration date through stability studies to analyze toxicity through stability studies and analyze toxicity through animal studies before gets marketed, There is a need to analyze it's properties according to the guidelines of regulatory bodies involving various statistical techniques cited in technical section of statistics in NDA. After the approval by the FDA it requires that drug product be tested for it's identity, strength quality and purity before it can be released into the market so there is a need to analyze and provide quality assurance of the final product. 


\section{BIAS AND VARIABILITY:}

For the approval of a drug regulatory agencies usually require results of studies conducted at various stages of drug research and development (R\&D) and provide a fair assessment for treatment of different ailments which together relate accuracy and reliability referred as closeness and degree of results to true value.

Any deviation of true value will lead to bias that occur due to false statistical procedures, The reliability of a study is an assessment of precision that measure degree of closeness to true value the higher the precision the more likely the results are reproducible.

\section{CONFOUNDING \& INTERACTION:}

During drug research and development(R\&D) there exists a different critical ailments that have impact on evaluation of treatment, These variations when not identified and controlled will deploy the potency of the drug, Therefore statistical interaction is employed to investigate whether the contribution of each factor when considered alone in practice show possible confounding results and interactions to be identified and controlled at different stages in order to ensure the constant effectiveness of the product.

\section{TYPE-I ERROR:}

Error is defined as a process or mistake that occurs during a process unknowingly they are divided into two types, i'e type- I\&II.

Type-I errors are serious and should take a limit the probability of committing this kind of error to a acceptable level, The acceptable level of type-I error is referred as significance level. A statistically significant result suggests that the null hypothesis be rejected in favour of alternative hypothesis, The probability of observing a type -I error is usually referred as P-value of test on the other hand probability of committing type-II error subtracted from type-I is called power of test.

\section{RANDOMIZATION:}

The interference on a parameter of interest of a population under study is usually derived under probability structure of parameter. Sampling is one of the methods where probability structure depends upon randomization which gives a negative impact on validity of probability structure, Therefore appropriate randomization methods under a valid model should be employed to ensure validity accuracy, reliability of statistical inference.

\section{Sample size determination:}

Sample size determination generally involves calculation of sample size for desired statistical properties such as precision, sample size justification etc. The objective is to determine whether the drug is effective an safe sample size can be determined/justified based on some criteria of type-I error or type-II error. In order to obtain the accurate result there need to be following certain criteria they are:-

- Study objective should be clearly stated

- A valid design with appropriate statistical tests should be used

- Sample size should be determined based on test of interest.

\section{Statistical difference \&scientific difference:}

It is defined as a difference that is unlikely to occur by chance alone, while a scientific difference is a tolerance that is considered to be scientific importance it is also called statistical significance.

\section{One sided v/s Two sided test:}

For the evaluation of drug product the null hypothesis of interest is often no difference the alternative hypothesis is usually there is a difference this type of test is called two sides test. Sometimes there exists difference against the alternative hypothesis that the drug is superior this the statistical test for this setting is called one side test. The pharmaceutical industry either uses a one sides or two sided test which has been challenged by several companies on drug efficacy \& study implementation (DESI) drugs.

\section{European statistics code of good practice:}

In February-2005 the statistical program committee has adopted the European statistics code of good practice and undertook to observe the fifteen principles established this code was embraced by (INEI).

1) Professional Independence

2) Mandate for data collection

3) Adequacy \& resources

4) Quality commitment

5) Statistical confidentially

6) Impartiality \& objectivity

7)Sound methodology

8) Appropriate statistical procedures

9) Nom excessive burden on respondents

\section{Cost effectiveness}

11Relevance

12Accuracy \& reliability

13Timeliness \& punctuality

14) Coherence \& compatibility

15) Accessibility \& clarity

Implementation of GSP:

The consideration of good statistical practice during the product research and development (R\&D) will effect the various ailments that define the properties to consolidate the characteristics according to various regulatory bodies,In the process of drug development there need to follow guidelines for effective and potent drug to be formulated these standards will describe the drug in all aspects that ensure safety of pharmaceutical product.

Communication and flexibility are important for the success of GSP.Improper/Inefficient communication between statisticians and pharmaceutical scientists or regulatory bodies will effect product development and failure in the statistical methods.The regulatory requirements of the drug development and approval process vary from drug to another drug therefore statistical coordinated advanced education should be provided for the efficacy of the pharmaceutical product. The enhancement of various techniques in different stages of product development will ensure a full-fledged potent pharmaceutical product. A panel 
discussion of various different disciplines to be hold together to evaluate the standards and the statistical principles for implementation of GSP.

\section{CONCLUSION:}

There exists various aspects of properties standards \& characteristics to be considered before marketing the pharmaceutical product which are maintained accordingly by guidance of formularies, regulatory bodies, standard operating procedures, GLP, GCP, CGMP, GRP, etc. The pharmaceutical product being manufactured should be formulated with utmost concern following all the regulatory standards principles to enhance the drug properties in terms of potency and efficacy this can only be achieved when there exists a healthy relation between the industry with regulatory bodies, statisticians, scientists.

\section{REFERENCES:}

1) Chow, S.C. Good statistics practice in drug development and regulatory approval process .Drug Inf.J.1997, 31;1157-1166.

2) Dubey S.D some thought on the one-sided and two -sided tests J. bio pharm stat 1991, 139-150.

3) ICH statistical principles for clinical trials, 1997.

4) USP/NF The United States pharmacopeia XXIV and the National formulary XIX, United states Pharmacopoeial convention, inc:Rockville,MD,2000.

5) Spriet,A,dupin-spriet, T. Good biometrics practice proposals for a setoff procedure Drug inf J.1992,26 405-409. 